\title{
INTEGRALS INVOLVING PRODUCTS OF BESSEL FUNCTIONS
}

\author{
by R. K. SAXENA
}

(Received 11 February, 1963)

1. Introductory. In this paper certain infinite integrals involving products of four Bessel functions of different arguments are evaluated in terms of Appell's function $F_{4}$ by the methods of the operational calculus. The results obtained are believed to be new.

As usual, the conventional notation $\phi(p) \doteqdot h(t)$ will be used to denote the classical Laplace integral relation

$$
\phi(p)=p \int_{0}^{\infty} e^{-p h} h(t) d t
$$

In the proofs of the formulae the following results will be required [1, pp. 281, 284], $[3$, pp. 78, 79].

$$
\exp \left(-\frac{\gamma+\delta}{p}\right) I_{\mu}\left(\frac{2 \sqrt{ }(\gamma \delta)}{p}\right) \doteqdot J_{\mu}(2 \sqrt{ }(\gamma t)) J_{\mu}(2 \sqrt{ }(\delta t))
$$

where $R(\mu)>-1$.

$$
\exp \left(\frac{\gamma+\delta}{p}\right) I_{\mu}\left(\frac{2 \sqrt{ }(\gamma \delta)}{p}\right) \doteqdot I_{\mu}(2 \sqrt{ }(\gamma t)) I_{\mu}(2 \sqrt{ }(\delta t))
$$

where $R(\mu)>-1$ and $R(p)>0$.

$$
p^{1-\lambda} e^{-\gamma / p} I_{\mu}(\gamma / p) \doteqdot \frac{\gamma^{\mu} t^{\lambda+\mu-1}}{2^{\mu} \Gamma(\mu+1) \Gamma(\lambda+\mu)}{ }_{1} F_{2}\left(\mu+\frac{1}{2} ; 2 \mu+1, \lambda+\mu ;-2 \gamma t\right)
$$

where $R(\lambda+\mu)>0$.

$$
2 p K_{v}(2 \sqrt{ }(\alpha p)) I_{v}(2 \sqrt{ }(\beta p)) \div \frac{1}{t} \exp \left(-\frac{\alpha+\beta}{t}\right) I_{\nu}\left(\frac{2 \sqrt{ }(\alpha \beta)}{t}\right)
$$

where $R(\alpha)>0, R(\beta)>0$ and $R(p)>0$.

$$
K_{v}(z)=\frac{1}{2} \sum_{v,-v} \Gamma(-v) \Gamma(1+v) I_{v}(z)
$$

and

$$
K_{-v}(z)=K_{v}(z)
$$


2. Integrals. The first of the integrals to be established here is

$$
\begin{aligned}
& \int_{0}^{\infty} t K_{v}(\alpha t) I_{\nu}(\beta t) J_{\mu}(\gamma t) J_{\mu}(\delta t) d t=\frac{(\alpha \beta)^{\nu}(\gamma \delta)^{\mu} \Gamma(\mu+v+1)}{\left(\alpha^{2}+\beta^{2}+\gamma^{2}+\delta^{2}\right)^{\mu+\nu+1} \Gamma(\nu+1) \Gamma(\mu+1)} \\
& \times F_{4}\left[\frac{\mu+\nu+1}{2}, \frac{\mu+v+2}{2} ; \nu+1, \mu+1 ; \frac{4 \alpha^{2} \beta^{2}}{\left(\alpha^{2}+\beta^{2}+\gamma^{2}+\delta^{2}\right)^{2}}, \frac{4 \gamma^{2} \delta^{2}}{\left(\alpha^{2}+\beta^{2}+\gamma^{2}+\delta^{2}\right)^{2}}\right]
\end{aligned}
$$

where $R(\mu+\nu+1)>0, R(\alpha)>|R(\beta)|+|\operatorname{Im} \gamma|+|\operatorname{Im} \delta|$.

To prove this we use the operational pairs (2) and (5) in the Goldstein form of the Parseval relation [2, p. 105] and then apply the formula [1, p. 196, (13)].

The following results can be derived in the same way from the pair of formulae (4), (5) and $(3),(5)$ respectively.

$$
\begin{aligned}
& \int_{0}^{\infty} t^{2 \lambda+2 \mu-1} K_{v}(\alpha t) I_{v}(\beta t){ }_{1} F_{2}\left(\mu+\frac{1}{2} ; 2 \mu+1, \lambda+\mu ;-\gamma^{2} t^{2}\right) d t \\
& =\frac{2^{2(\lambda+\mu-1)}(\alpha \beta)^{v} \Gamma(\lambda+\mu+v) \Gamma(\lambda+\mu)}{\left(\alpha^{2}+\beta^{2}+2 \gamma^{2}\right)^{\lambda+\mu+v} \Gamma(v+1)} \\
& \quad \times F_{4}\left[\frac{\lambda+\mu+v}{2}, \frac{\lambda+\mu+v+1}{2} ; v+1, \mu+1 ; \frac{4 \alpha^{2} \beta^{2}}{\left(\alpha^{2}+\beta^{2}+2 \gamma^{2}\right)^{2}}, \frac{4 \gamma^{4}}{\left(\alpha^{2}+\beta^{2}+2 \gamma^{2}\right)^{2}}\right],
\end{aligned}
$$

where $R(\lambda+\mu+v)>0, R(\lambda+\mu)>0$ and $R(\alpha)>|R(\beta)|+|\operatorname{Im} \gamma|$.

$$
\begin{aligned}
& \int_{0}^{\infty} t K_{v}(\alpha t) I_{v}(\beta t) I_{\mu}(\gamma t) I_{\mu}(\delta t) d t=\frac{(\alpha \beta)^{v}(\gamma \delta)^{\mu} \Gamma(\mu+v+1)}{\left(\alpha^{2}+\beta^{2}-\gamma^{2}-\delta^{2}\right)^{\mu+v+1} \Gamma(v+1) \Gamma(\mu+1)} \\
& \quad \times F_{4}\left[\frac{\mu+\nu+1}{2}, \frac{\mu+\nu+2}{2} ; v+1, \mu+1 ; \frac{4 \alpha^{2} \beta^{2}}{\left(\alpha^{2}+\beta^{2}-\gamma^{2}-\delta^{2}\right)^{2}}, \frac{4 \gamma^{2} \delta^{2}}{\left(\alpha^{2}+\beta^{2}-\gamma^{2}-\delta^{2}\right)^{2}}\right],
\end{aligned}
$$

where $R(\mu+v+1)>0, R(\mu+1)>0$ and $R(\alpha)>|R(\beta)|+|R(\gamma)|+|R(\delta)|$.

On applying (6) to (8), (9) and (10) and using (7), we find that

$$
\begin{aligned}
& \int_{0}^{\infty} t K_{v}(\alpha t) K_{v}(\beta t) J_{\mu}(\gamma t) J_{\mu}(\delta t) d t=\frac{(\gamma \delta)^{\mu}}{2 \Gamma(\mu+1)} \sum_{\nu,-v} \frac{(\alpha \beta)^{v} \Gamma(-v) \Gamma(\mu+v+1)}{\left(\alpha^{2}+\beta^{2}+\gamma^{2}+\delta^{2}\right)^{\mu+v+1}} \\
& \times F_{4}\left[\frac{\mu+\nu+1}{2}, \frac{\mu+v+2}{2} ; v+1, \mu+1 ; \frac{4 \alpha^{2} \beta^{2}}{\left(\alpha^{2}+\beta^{2}+\gamma^{2}+\delta^{2}\right)^{2}}, \frac{4 \gamma^{2} \delta^{2}}{\left(\alpha^{2}+\beta^{2}+\gamma^{2}+\delta^{2}\right)^{2}}\right]
\end{aligned}
$$

where $R(1+\mu \pm v)>0, R(\alpha+\beta)>|\operatorname{Im} \gamma|+|\operatorname{Im} \delta|$; 


$$
\begin{aligned}
& \int_{0}^{\infty} t^{2 \lambda+2 \mu-1} K_{v}(\alpha t) K_{v}(\beta t){ }_{1} F_{2}\left(\mu+\frac{1}{2} ; 2 \mu+1, \lambda+\mu ;-\gamma^{2} t^{2}\right) d t \\
& =2^{2 \lambda+2 \mu-3} \Gamma(\lambda+\mu) \sum_{v_{v}-v} \frac{(\alpha \beta)^{\nu} \Gamma(-v) \Gamma(\lambda+\mu+v)}{\left(\alpha^{2}+\beta^{2}+2 \gamma^{2}\right)^{\lambda+\mu+\nu}} \\
& \quad \times F_{4}\left[\frac{\lambda+\mu+v}{2}, \frac{\lambda+\mu+v+1}{2} ; v+1, \mu+1 ; \frac{4 \alpha^{2} \beta^{2}}{\left(\alpha^{2}+\beta^{2}+2 \gamma^{2}\right)^{2}}, \frac{4 \gamma^{4}}{\left(\alpha^{2}+\beta^{2}+2 \gamma^{2}\right)^{2}}\right],
\end{aligned}
$$

where $R(\lambda+\mu \pm v)>0, R(\alpha+\beta)>|\operatorname{Im} \gamma|$; and

$$
\begin{aligned}
\int_{0}^{\infty} t K_{v}(\alpha t) K_{v}(\beta t) I_{\mu}(\gamma t) I_{\mu}(\delta t) d t=\frac{(\gamma \delta)^{\mu}}{2 \Gamma(\mu+1)} \sum_{\nu,-v} \frac{(\alpha \beta)^{v} \Gamma(-v) \Gamma(\mu+v+1)}{\left(\alpha^{2}+\beta^{2}-\gamma^{2}-\delta^{2}\right)^{\mu+v+1}} \\
\times F_{4}\left[\frac{\mu+\nu+1}{2}, \frac{\mu+v+2}{2} ; v+1, \mu+1 ; \frac{4 \alpha^{2} \beta^{2}}{\left(\alpha^{2}+\beta^{2}-\gamma^{2}-\delta^{2}\right)^{2}}, \frac{4 \gamma^{2} \delta^{2}}{\left(\alpha^{2}+\beta^{2}-\gamma^{2}-\delta^{2}\right)^{2}}\right],
\end{aligned}
$$

where $R(1+\mu \pm v)>0, R(\alpha+\beta)>|R(\gamma)|+|R(\delta)|$.

\section{REFERENCES}

1. A. Erdélyi et al., Tables of integral transforms, Vol. I (New York, 1954).

2. S. Goldstein, Operational representation of Whittaker's confluent hypergeometric function and Weber's parabolic cylinder functions, Proc. London Math. Soc. 34 (1932), 103-125.

3. G. N. Watson, Theory of Bessel functions (Cambridge, 1922).

UNIVERSITY OF JODHPUR JODHPUR, INDIA 